\title{
miR-132 mediates cell permeability and migration by targeting occludin in high-glucose -induced ARPE-19 cells
}

\author{
Shan-Shan Wang, Xing Liao, Fei Liu, Qian Zhang, Jing-Jing Qiu and Shu-Hua Fu \\ Department of Ophthalmology, The Second Affiliated Hospital of Nanchang University, Nanchang 330006, P. R. China
}

\begin{abstract}
This study investigated the effects and mechanisms of miR-132 related to the permeability and mobility of human retinal pigment epithelium ARPE-19 cells in high-glucose (HG) condition. ARPE-19 cells were cultured in normal and HG condition and identified by immunofluorescence staining. Cell viability was assessed by the MTT assay, cell permeability was assessed by the FITC-dextran assay and cell mobility was assessed by the wound healing assay. Different miRNA and mRNA expression levels were determined by quantitative real-time polymerase chain reaction (RT-qPCR). The expression of tight junction-related proteins was determined by Western blot assay and immunofluorescence. The interaction between occludin and miR-132 was confirmed by a dual-luciferase reporter assay. We revealed that HG-treated ARPE-19 cells exhibited significantly increased miR-132 expression, decreased expression of the tight-junction markers including occludin and Ecadherin, and increased cell mobility and permeability. Occludin is a direct target of miR-132, which could regulate cell viability, mobility and permeability under HG condition through the JAK/STAT3 signaling pathway. These are the first data to suggest that miR-132 may contribute to the progression of diabetic retinopathy (DR) and that targeting the effect of miR-132 on occudin and the JAK/STAT3 pathway could represent a novel effective DR-treatment strategy.
\end{abstract}

Key words: Diabetic retinopathy, JAK/STAT3 pathway, miR-132, Occludin

AS ONE of the most serious and common chronic eye complications of diabetes, diabetic retinopathy (DR) has become the leading cause of blindness in adults $[1,2]$. Progressive loss of vascular cells and slow dissolution of inter-vascular junctions are the main characteristics of DR [3]. DR is associated with diabetic macular edema (DME), and it presents microvascular and macrovascular changes [4]. Human retinal pigment epithelial cells, components of the blood-retinal barrier (BRB), could be impaired by the adverse effects of high glucose (HG) in the early stage of this disease, which induces dysfunction of the BRB, thus contributing to progression of DR $[5,6]$. Recently, to elucidate the mechanism of DR, significant efforts $[7,8]$ have been focused on vascular endothelial growth factor (VEGF) [9], defects of which contribute prominently to the clinical features of $\mathrm{DR}$, and have led to the inference of the general microvascular nature of this diabetic complication[10, 11].

Submitted May 15, 2020; Accepted Dec. 2, 2020 as EJ20-0277 Released online in J-STAGE as advance publication Feb. 9, 2021 Correspondence to: Shu-Hua Fu, Department of Ophthalmology, The Second Affiliated Hospital of Nanchang University, No. 1, Minde Road, Nanchang 330006, Jiangxi Province, P. R. China.

E-mail: fushua.student@sina.com
However, there is still little known about the molecular mechanisms involved in increased retinal vascular permeability.

At present, miRNAs have been reported to play pivotal roles in the regulation of apoptosis, proliferation and migration of retinal cells and participate in regulating DR-related neovascularization [12]. A number of studies have investigated the roles of miRNAs in DR using HRECs and RPE cells, and in vivo models of streptozotocin (STZ)-induced diabetic retinas. In addition, they have identified a series of miRNAs whose expression is altered in the retinas and retinal endothelial cells (RECs) of STZ-induced diabetic rats 3 months after the onset of diabetes [13]. In microvascular endothelial cells (ECs), miR-200b, which directly targeted VEGF, was decreased after treatment with HG [14]. miR-146 was first implicated in DR and can modulate the NF- $\kappa B$ inflammatory response that played a crucial role in the early phases of DR development [15]. miR-126, miR-29b and miR-195 were also reported to play roles in the DR process. Moreover, aberrant miRNAs could be biomarkers in the serum of patients with DR [16], and six miRNAs were discovered to be significantly overexpressed and related to fibrosis and angiogenesis in DR [17]. 
Occludin is a protein of the claudin group and the key component of tight junction [18], playing a pivotal role in maintaining their barrier properties. Therefore, absence or mutation of occludin could increase epithelial leakiness, which is a critical barrier to cancer prevention [19], and it can also cause reduced adhesion and tight junction function and increased invasion in breast cancer tissues [20]. In addition, occludin participates in the regulation of apoptosis, disruption of which is an important aspect of a number of diseases [21]. It has been reported that tight junction abnormalities resulting from its disruption are closely associated with the progression of retinal pathology in DR. Reduced expression of occludin has been implicated in the breakdown of the BRB [22]. Compromised tight junction in endothelial cells was also found to be present on the retinas of diabetic rats [23, 24] and those of diabetic patients $[25,26]$. Thus, we infer that preventing or reversing the downregulation of occludin may be a potential therapeutic target for DR. Moreover, recent studies have shown that occludin is also the key factor through which the JAK/STAT3 pathway may regulate the process of epithelial-mesenchymal transition (EMT) [27]. Whether this pathway is involved in occludin in the process of DR needs further investigation.

In this study, we found that miR-132 is one of the miRNAs targeting occludin through bioinformatics analysis, and we provide the first evidence of the regulatory role of miR-132 in retinal barrier function in DR through targeting occludin and activating the JAK/STAT3 pathway, suggesting a promising alternative therapeutic strategy for treating vascular permeability in DR.

\section{Materials and Methods}

\section{Cell culture}

The human retinal pigmented epithelium cell line ARPE-19 was obtained from the American Type Culture Collection (ATCC, Manassas, VA). Cells were cultured in Dulbecco's modified Eagle's medium (DMEM) plus $10 \%$ fetal bovine serum (FBS, GIBCO, CA) at $37^{\circ} \mathrm{C}$ with $5 \% \mathrm{CO}_{2}$. For $\mathrm{HG}$ treatment, the ARPE-19 cells were exposed to D-glucose (Sigma) ata concentration of $30 \mathrm{mmol} / \mathrm{L}$ in cultures as described in previous publications [28]. For normal glucose treatment, ARPE-19 cells were exposed to D-glucose $(5.6 \mathrm{mmol} / \mathrm{L})$ in cultures. For hypertonic treatment, ARPE-19 cells were exposed to D-glucose $(5.6 \mathrm{mmol} / \mathrm{L}$ ) and D-mannitol (Sigma, $24.4 \mathrm{mmol} / \mathrm{L}$ ) in cultures.

\section{Immunofluorescence}

Permeable Transwell filters were used to seed ARPE-19 cells. Cells were fixed and permeabilized in $100 \%$ ethanol for $20 \mathrm{~min}$ at $-20^{\circ} \mathrm{C}$. Then, PBS contain- ing $1 \%$ bovine serum albumin (blocking buffer) was used to block cells for $1 \mathrm{~h}$ at room temperature (RT). Primary antibodies were cultured overnight in a 1:250 dilution (mouse anti-keratin $0.25 \mathrm{mg} / \mathrm{mL}$; mouse anti-Ecadherin $0.25 \mathrm{mg} / \mathrm{mL}$; mouse anti-Vimentin 0.25 $\mathrm{mg} / \mathrm{mL}$; mouse anti-occludin $0.25 \mathrm{mg} / \mathrm{mL}$ ). All the antibodies were purchased from Zymed, San Francisco, USA. Coverslips were then washed three times with PBS the next day. Corresponding secondary fluorophorelabelled antibodies in PBS/1\% BSA were then added and incubated for $60 \mathrm{~min}$ at room temperature. Cells were washed twice with PBS and mounted in phenylethylamine and were analyzed with a Zeiss LSM510 laser scanning confocal microscope (Zeiss, Germany).

\section{Cell transfection}

The miR-132 mimics and inhibitors and the scrambled negative control (si-NC, NC mimics and $\mathrm{NC}$ inhibitor) were purchased from Shanghai GenePharma, China. The pcDNA3.1-occludin and pcDNA3.1 empty vector (Vector) were purchased from Generay (Shanghai, China). Transfections were performed with Lipofectamine 2000 (Invitrogen, USA) according to the manufacturer's instructions. We optimized the concentration of transfection reagents to permit the highest transfection efficiency with the least cell death $(<10 \%)$.

\section{Quantitative real-time polymerase chain reaction (RT-qPCR)}

TRIzol (Invitrogen, USA) was used to isolate total RNA. Then, to generate first-strand cDNA, the ImPromII Reverse Transcription System (Promega, USA) and random primers or Q-miRT primers were used [29]. We then carried out a RT-qPCR test of mRNA with SYBR Premix Ex Taq (TaKaRa, Japan) and gene-specific primers. GAPDH was used for normalization. RT-qPCR for microRNA analysis was performed as described before [29] and U6 snRNA was employed as an endogenous control. The comparative $\mathrm{Ct}$ method was carried out to calculate the relative expression levels of RNAs. Primers used for real-time PCR were as follows: E-cadherin-F: 5'-GCCCCATCAGGCCTCCGTTT-3', E-cadherin-R: 5'ACCTTGCCTTCTTTGTCTTTGTTGGA-3'. VimentinF: 5'-CCTGAACCTGAGGGAAACTAA-3', VimentinR: 5'-GCAGAAAGGCACTTGAAAGC-3'. occludin-F: 5'-CCAAGGTTCCATCCGAAGCA-3', occludin-R: 5'-G CGCTGTTAAAGGAACCGC-3'. hsa-miR-425: 5'-AAT GACACGATCACTCCCGTTGA-3'. hsa-miR-383: 5'-A GATCAGAAGGTGATTGTGGCT-3'. hsa-miR-7: 5'-TG GAAGACTAGTGATTTTGTTGTT-3'. hsa-miR-132: 5'TAACAGTCTACAGCCATGGTCG-3'. hsa-miR-212: 5'-TAACAGTCTCCAGTCACGGCC-3'. GAPDH-F: 5'TCAGTGGTGGACCTGACCTG-3', GAPDH-R: 5'-TG 
CTGTAGCCAAATTCGTTG-3'. U6-F： 5'-CGCTTCGG CAGCACATATACTA-3', U6-R: 5'-CGCTTCACGAATT TGCGTGTCA-3'.

\section{Cell migration assay}

Cells were scratched to create a wound with a $1 \mathrm{~mm}$ initial width and cultured under normal condition. We measured the migrated distances at $48 \mathrm{~h}$ after scratching.

\section{MTT assay}

After culturing for 2 days, cell viability of ARPE-19 cells was determined with a colorimetric MTT assay kit (Sigma, USA) following the instructions of the kit. Absorbance at $490 \mathrm{~nm}$ was determined using a microplate reader.

\section{Cell permeability measurement}

FITC-dextran was used to measure ARPE-19 cell permeability. $10 \%$ chloral hydrate was used to anaesthetized the cultured cells and FITC-dextran $(1 \mathrm{~mL}, 50 \mathrm{mg} / \mathrm{mL}$, dissolved in sterile $0.9 \% \mathrm{NaCl}$ solution) was then added for $10 \mathrm{~min}$. Cells were soaked in $4 \%$ paraformaldehyde solution for $24 \mathrm{~h}$ at $4^{\circ} \mathrm{C}$. After that, Cells were then embedded with optimal cutting temperature (OCT) compound. Finally, a microplate reader (Gemini ${ }^{\circledR} \mathrm{EM}$, Molecular Devices, USA) was used to detect cell permeability.

\section{Western-blot analysis}

IP lysis buffer (Pierce, USA) containing protease inhibitors (Sigma, USA) was used to lyse cells. Equal amounts of protein were then separated by $10 \%$ SDSPAGE after BCA (Pierce, USA) quantification, electrophoretically transferred to nitrocellulose membrane (Millipore, USA), and incubated with specific primary antibodies $(1: 1,000)$ followed by corresponding secondary antibodies $(1: 5,000)$ (Pierce, USA). Chemiluminescent substrate (Millipore, USA) was then used for signal visualization and film exposure. Occludin, E-cadherin, Vimentin, JAK, STAT3 (Santa Cruz Biotechnology, USA); p-JAK, p-STAT3 (CST, USA); and GAPDH (Proteintech, USA) were used for this assay.

\section{Dual-luciferase reporter assay}

The fragments from the 3'-untranslated region (3'UTR) of occludin containing predicted miR-132 binding sites and their mutant sequences were sub-cloned to the pmirGLO plasmids (Promega, USA) to form occludin wild-type (WT-occludin), and occludin mutated-type (MUT-occludin). miR-132 mimics (50 pmol/well) and luciferase reporter plasmids ( $800 \mathrm{ng} /$ well) were cotransfected into HEK-293T cells by Lipofectamine 3000 (Invitrogen, USA). Then, the relative luciferase activities were measured with a dual-luciferase assay system (Promega, USA) $48 \mathrm{~h}$ after transfection.

\section{Statistical analysis}

Data are presented as the mean \pm SD from at least three independent experiments. One-way analysis of variance (one-way ANOVA) was used for multiple comparisons to assess the significant differences between groups. Student's $t$-test was performed for comparisons between two groups. Statistical analyses were performed using GraphPad Prism 6.0 (GraphPad Prism, USA). $p<0.05$ was considered statistically significant.

\section{Results}

\section{High-glucose treatment alters ARPE-19 cell viability, mobility and permeability}

After 3 days of cell culture, the cells attached to the vessel wall to form abundant proliferating colonies. The proliferating cells were deformed into polygons, and the un-proliferated cells were bark-shaped. After one week of cell culture, the monolayer cells tended to fuse, and the morphology was uniform, with a polygonal paving stone appearance and a central nucleus. After immunocytochemical staining of ARPE-19 cells (light microscopy) with a cytokeratin antibody, the cytoplasm of cultured ARPE-19 cells had a red coloration, and keratin expression was positive (Fig. 1A, top). ARPE-19 cells were then treated with $\mathrm{HG}$ to create an in vitro model of DR. The group with normal glucose treatment was used as a control. Optical microscopy was used to observe cell morphology within different groups. Compared with the normal glucose treatment group, cells in the hypertonic group had larger size with a normal morphology, while cells in the HG group had a slender form and scattered arrangement (Fig. 1A, bottom). The MTT assay showed that cells in the HG group had lower viability compared with that of the other two groups (Fig. 1B). E-cadherin, Vimentin and occludin are protein markers that are associated with tight junction. We analyzed their mRNA and protein expression by RT-qPCR, Western-blot and immunofluorescence. These analyses showed that E-cadherin and occludin were downregulated under HG treatment while Vimentin showed an opposite effect (Fig. 1C-E), which indicated that the tight junction of cells was impaired by HG. Moreover, the wound healing assay showed that cell migration was significantly induced by HG (Fig. 1F), and the FITC-dextran assay revealed that cell permeability was greatly increased in the HG group compared with that of the normal glucose and hypertonic groups (Fig. 1G). 
A

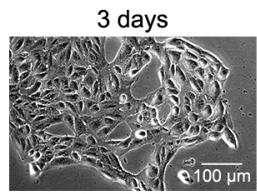

Control
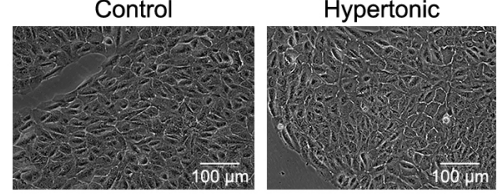

7 days

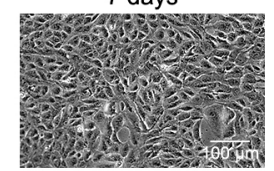

HG

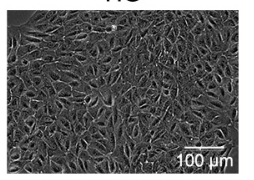

B

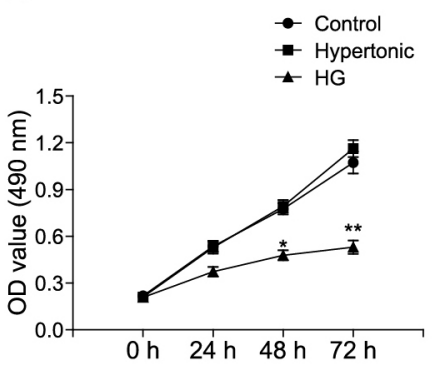

$E$

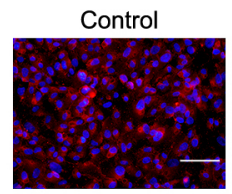

E-cadherin
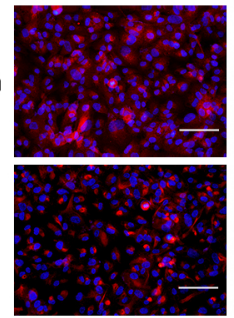

C

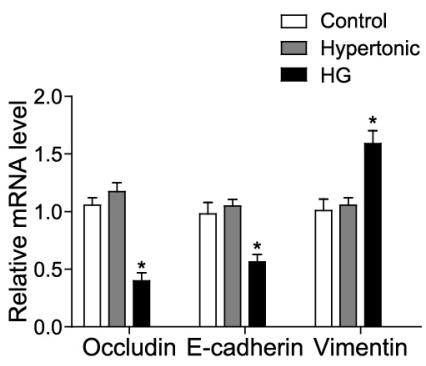

Hypertonic

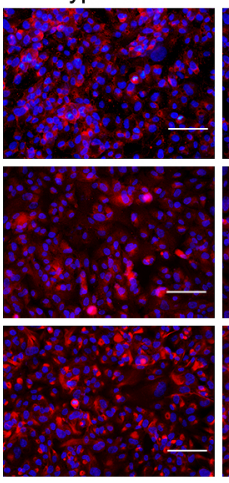

HG

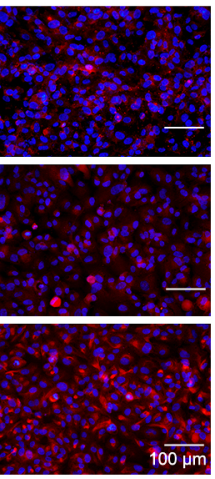

$\mathrm{F}$
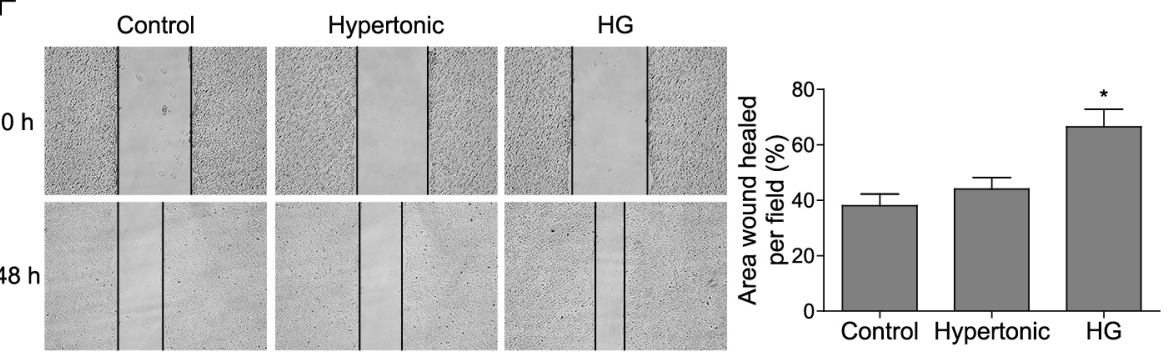

G

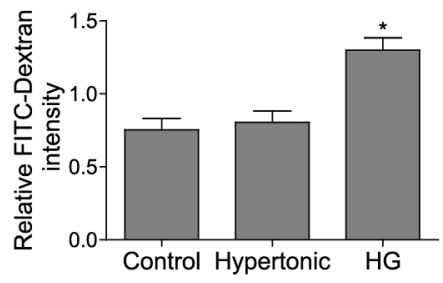

Fig. 1 High-glucose treatment regulated ARPE-19 cell viability, mobility and permeability

A. Morphology of cells was observed by optical microscopy, and ARPE-19 cells were identified by immunocytochemical staining. B. Viability of cells was analysed by the MTT assay. C-E. RT-qPCR, Western blot analysis and immunofluorescence of Ecadherin, Vimentin and occludin. RNA and protein levels were normalized to GAPDH. F. Cell migration ability changes were detected by the wound healing assay. G. FITC-dextran assay to detect cell permeability of different groups of cells. Data are presented as the mean \pm standard error (SD) $(n=3) .{ }^{*} p<0.05,{ }^{* *} p<0.01 v s$. normal glucose control group.

\section{MiR-132 is upregulated in ARPE-19 cells under high glucose condition and targets occludin}

To identify miRNAs that could target occludin, we searched published studies of changes in miRNAs expression in DR [13]. 5 miRNAs, including miR-132, miR-383, miR-425, miR-7 and miR-212, were selected. RT-qPCR analysis was then applied to identify which miRNAs could be upregulated in ARPE-19 cells by HG treatment. As shown in Fig. 2A, miR-132, miR-212 and miR-7 were upregulated in the HG condition, while miR-383 and miR-425 did not show a significant change. In addition, no changes in the expression levels of the 5 miRNAs were observed in the hypertonic group. Of the three upregulated miRNAs, miR-132 expression was increased the most, and therefore we chose it to investigate its function in the regulation of DR. In addition, we detected the expression of miR-132 at different time points (day 1, 3, 5 and 7). Fig. 2B shows that miR-132 expression was increased with the prolongation of $\mathrm{HG}$ treatment time $(p<0.05)$, and its expression was significantly increased at day 7 , to almost 8 times that of the normal control group $(p<0.001)$. However, no changes in the expression level of miR-132 were observed in the hypertonic group. In addition, TargetScan (http:// www.targetscan.org) was used to predict the binding site between miR-132 and occludin, and the dual-luciferase 

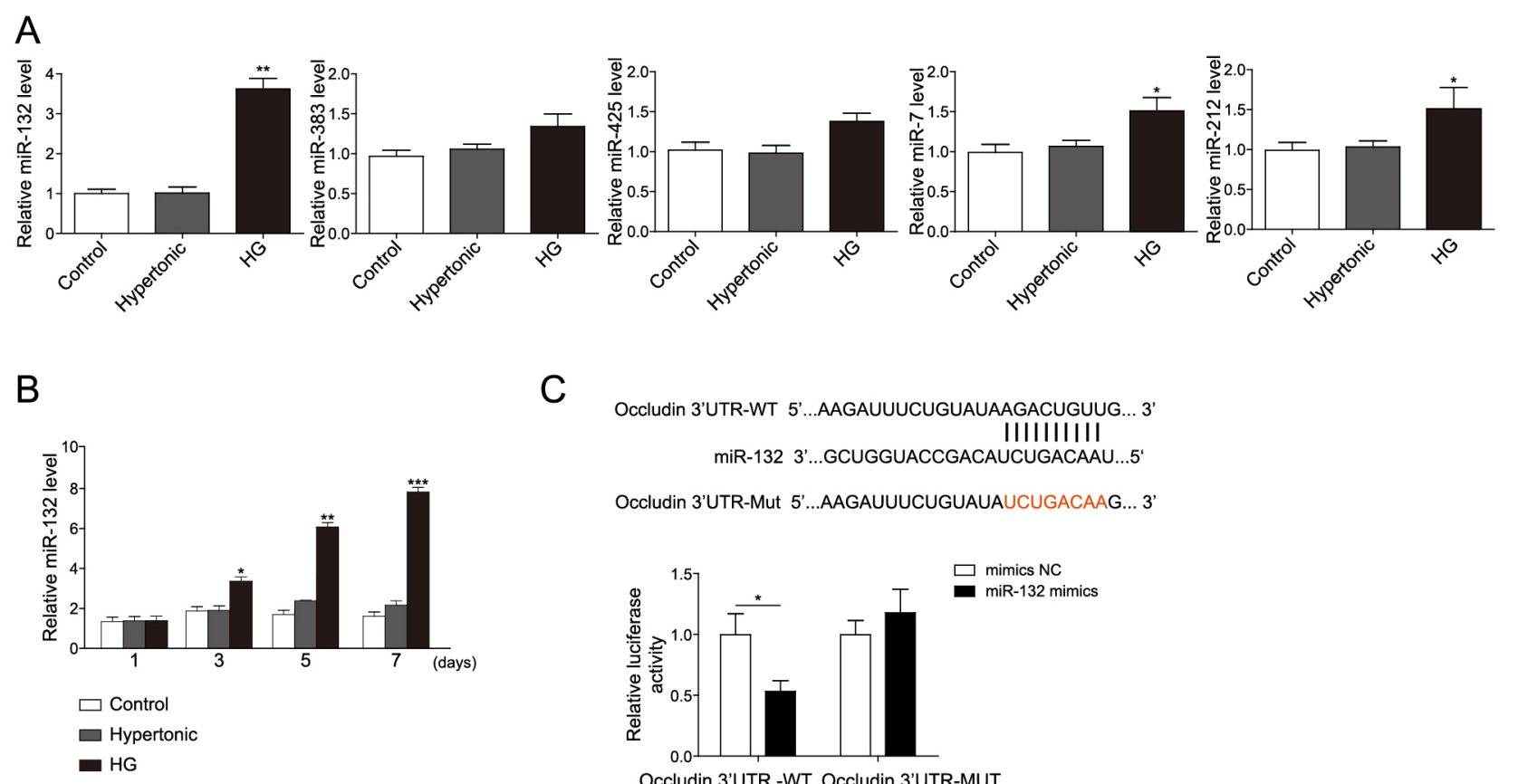

C
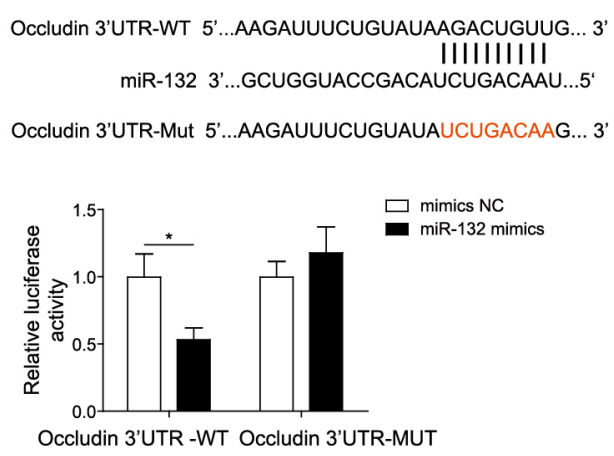

Fig. 2 miR-132 was upregulated in ARPE-19 cells under high-glucose condition and targets occludin

A. RT-qPCR analysis to detect the expression of miR-132, miR-383, miR-425, miR-7 and miR-212 under different treatments. B. Detection of miR-132 expression level over the time (day 1, 3, 5 and 7) by RT-qPCR. C. Prediction of the binding between miR-132 and the 3'-UTR of occludin mRNA was performed by bioinformatics analysis using TargetScan and confirmed by the dual-luciferase reporter assay. Data are presented as the mean \pm standard error (SD) $(n=3)$. $* p<0.05, * * p<0.01, * * * p<0.001$ vs. normal glucose control group.

reporter assay was used to confirm the relationship. The results showed that there is a binding site for miR-132 in the 3'-untranlational region (UTR) of occludin. Moreover, miR-132 mimics can significantly reduce the luciferase activity of tranfection with wild-type 3'UTR occludin, but there is not significant effect of tranfection with mutant type 3'UTR occludin (Fig. 2C). Taken together, there is a direct targeting relationship between miR-132 and occludin.

\section{miR-132 alters cell viability, mobility, and permeability under high glucose condition}

To investigate the role of miR-132 in DR, miR-132 mimics and $\mathrm{NC}$ mimics were transfected into ARPE-19 cells. Compared with the NC mimics group, miR-132 was upregulated in the miR-132 mimics group (Fig. 3A) and the miR-132 mimics group showed lower viability compared with the $\mathrm{NC}$ mimics group under HG condition (Fig. 3B), the mRNA and protein levels of occludin and E-cadherin were downregulated by miR-132 mimics, while the mRNA and protein levels of Vimentin were upregulated (Fig. 3C-D), which indicated that tight junction of cells was decreased by miR-132 mimics. Moreover, the wound healing assay showed that cell migration was significantly increased by miR-132 mimics (Fig. 3E). Meanwhile, the FITC-dextran assay also revealed that cell permeability was greatly increased (Fig. 3F). Therefore, by integrating the results above, it can be inferred that miR-132 could regulate cell viability, mobility and permeability under HG condition.

To further reveal the function of miR-132 and assess whether inhibiting the miR-132 level in ARPE-19 could rescue HG-induced perturbation of cell viability, cell mobility and permeability, miR-132 inhibitor and inhibitor NC were transfected into ARPE-19 cells. First, we evaluated the expression of miR-132 by RT-qPCR to verify the inhibitory effect, and the results demonstrated the level of miR-132 was downregulated after transfection with miR-132 inhibitor (Fig. 4A). Then, cell viability, mobility, and permeability were assessed. Compared with the inhibitor NC group, cells in the miR-132 inhibitor group had higher viability under $\mathrm{HG}$ condition (Fig. 4B). Both the mRNA and protein levels of occludin and $\mathrm{E}$-cadherin were upregulated by miR-132 inhibitor, while the mRNA and protein levels of Vimentin were downregulated (Fig. 4C-D), indicating that tight junction of cells was enhanced by inhibiting miR-132 under HG condition. Moreover, the wound healing assay showed that cell migration was significantly decreased by miR-132 inhibitor (Fig. 4E), and the FITC-dextran assay also revealed that cell permeability was greatly suppressed in the miR-132 inhibitor group compared to the 

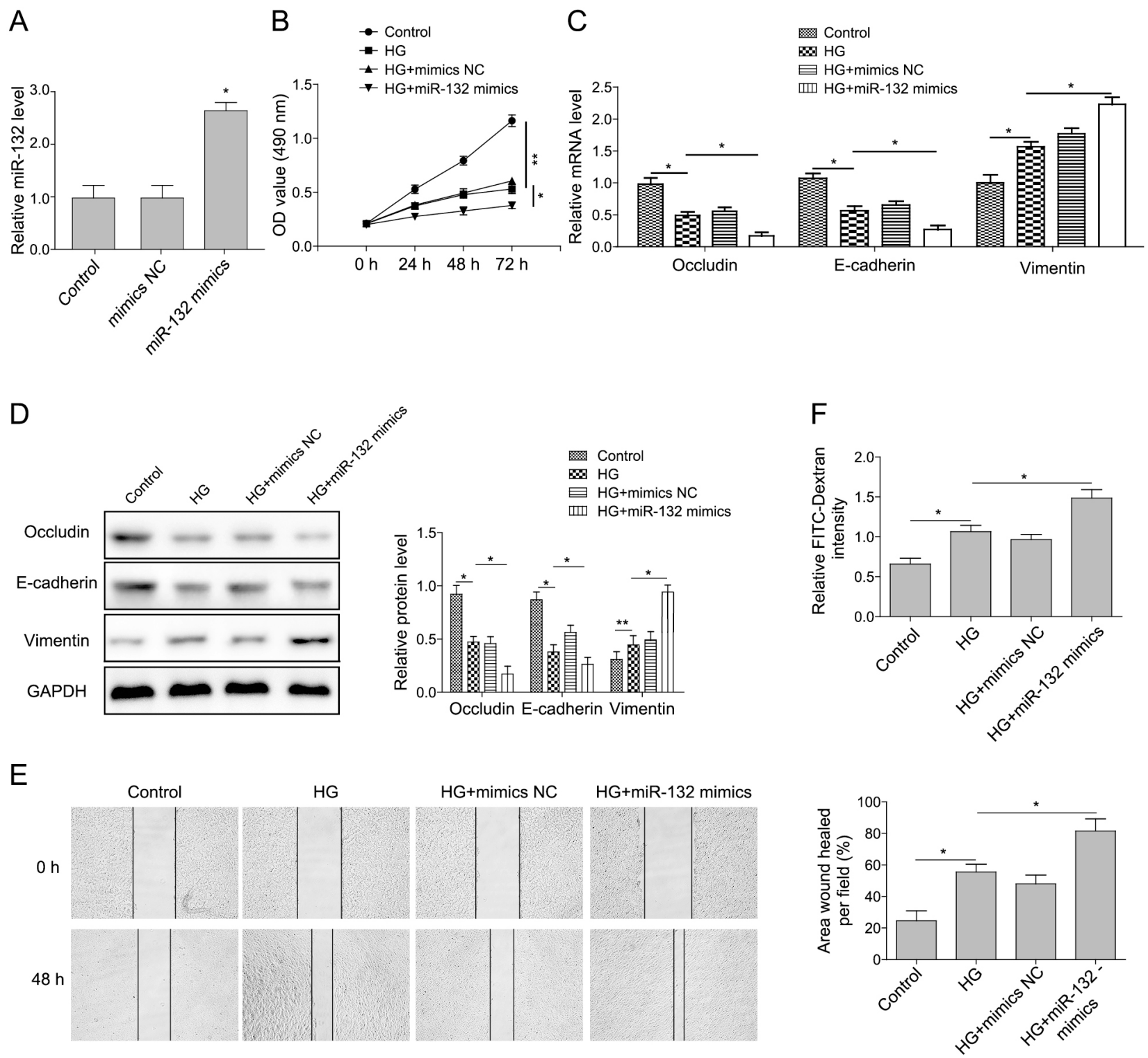

Fig. 3 MiR-132 overexpression downregulated cell viability and upregulated cell mobility and permeability of ARPE-19 cells under HG condition

A. RT-qPCR analysis to detect the expression of miR-132 after transfected with miR-132 mimics for $72 \mathrm{~h}$. B. Viability of cells under different treatments was analyzed by the MTT assay. C-D. RT-qPCR and Western blot analysis of occludin, E-cadherin and Vimentin. RNA and protein levels were normalized to GAPDH. E. Cell migration changes were detected by the wound healing assay. F. FITC-dextran assay to detect cell permeability of different groups of cells. Data are presented as the mean \pm standard error (SD) $(n=3) . * p<0.05, * * p<0.01$.

inhibitor NC group under HG condition (Fig. 4F, $p<$ 0.01 ). We also detected the expression of miR-132 in normal glucose condition or hypertonic condition and determined the viability and tight junction related gene expression after ARPE-19 cells were transfected with miR-132 mimics. The results showed that compared with the blank control group, the expression of miR-132 under normal glucose condition or hypertonic condition did not change significantly (Fig. 4G), and after overexpression of miR-132 in the normal glucose condition or hypertonic condition, the proliferation and the expression of tight junction related genes in ARPE-19 cells did not change significantly (Fig. 4H and 4I).

miR-132 activates the JAK/STAT3 signaling pathway through the regulation of occludin in ARPE-19 cells under high glucose condition

To investigate the downstream molecular mechanism of miR-132, ARPE-19 cells were then transfected with an occludin-overexpressing vector, and the results 

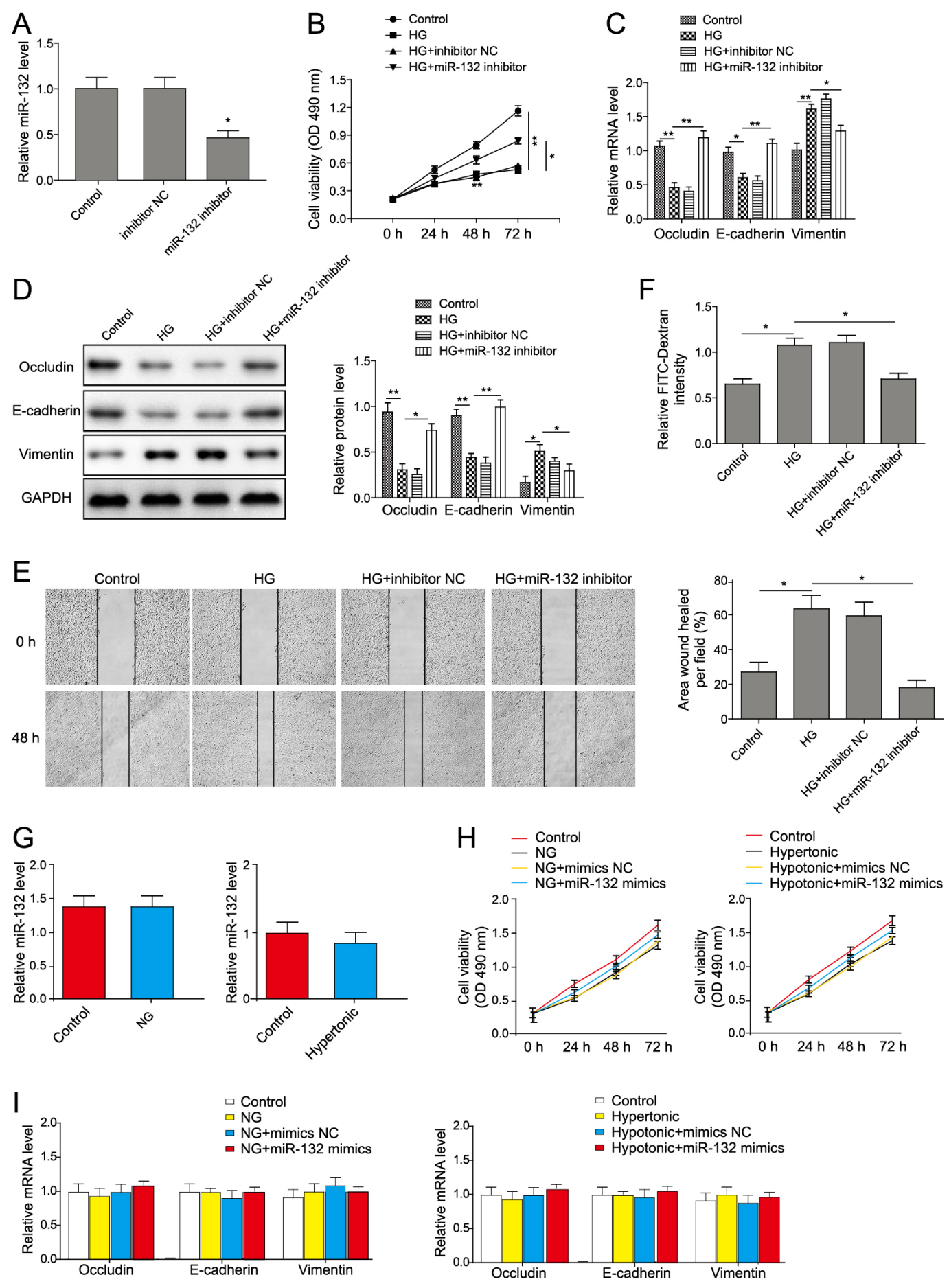

Fig. 4 MiR-132 knockdown upregulated cell viability and downregulated cell mobility and permeability under high-glucose condition A. RT-qPCR analysis to detect the expression of miR-132 after transfected with miR-132 inhibitor for $72 \mathrm{~h}$. B. Viability of cells under different treatments was analysed by the MTT assay. C-D. RT-qPCR and Western blot analysis of occludin, E-cadherin and Vimentin. RNA and protein levels were normalized to GAPDH. E. Cell migration changes were detected by the wound healing assay. F. FITC-dextran assay to detect cell permeability of different groups of cells. G. RT-qPCR analysis to detect the expression of miR-132 in ARPE-19 cells under normal glucose condition or or hypertonic condition. H. Viability of cells transfected with miR-132 mimics (mimics NC) under normal glucose condition or hypertonic condition was analysed by the MTT essay. I. RTqPCR analysis of occludin, E-cadherin and Vimentin of ARPE-19 cells transfected with miR-132 mimics (mimics-NC) under normal condition or hypertonic condition. RNA levels were normalized to GAPDH. Data are presented as the mean \pm standard error (SD) $(n=3) . * p<0.05, * * p<0.01$. normal glucose, $\mathrm{NG}$. 
A

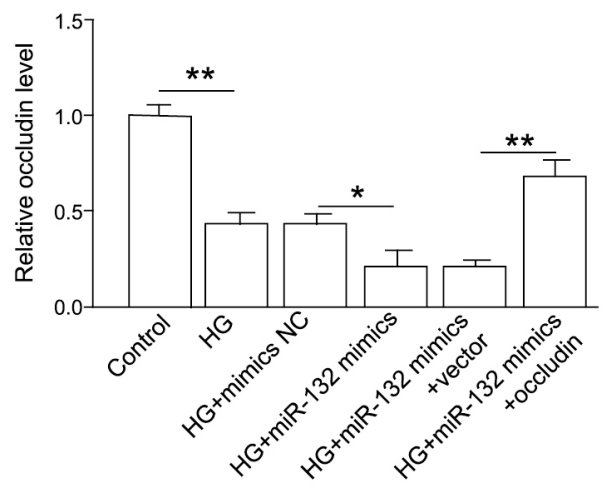

B

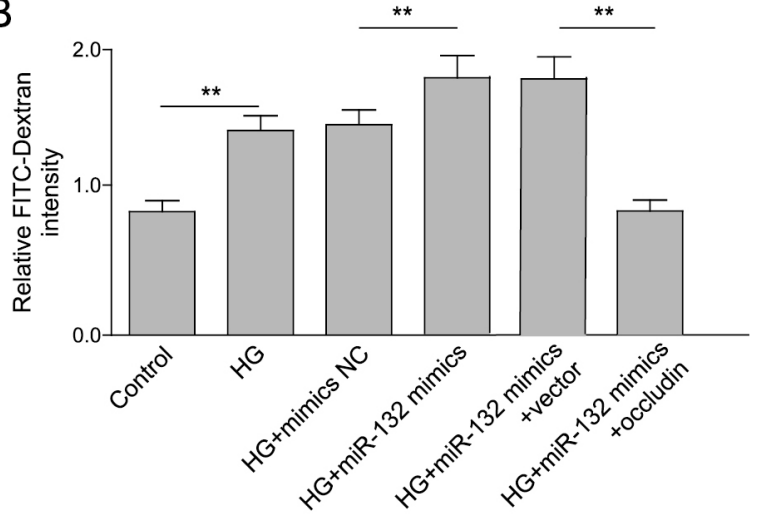

D

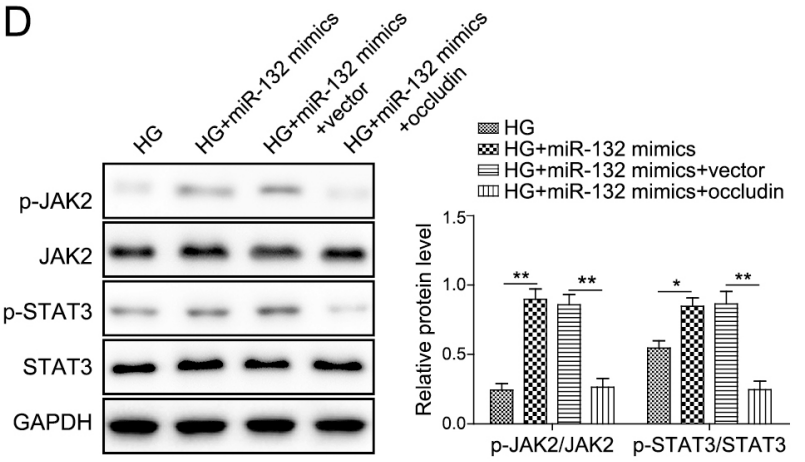

Fig. 5 miR-132 activated the JAK/STAT3 signalling pathway through regulation of occludin in ARPE-19 cells under high-glucose condition

A. RT-qPCR analysis to detect the expression level of occludin after transfected with miR-132 mimics and/or occludingoverexpressing vectors under HG condition for $72 \mathrm{~h}$. B. FITC-dextran assay to detect cell permeability after transfected with miR-132 mimics and/or occludin-overexpressing vectors under HG condition for $72 \mathrm{~h}$. C.Western blot analysis of p-JAK2 and pSTAT3 of ARPE-19 cells co-transfection with miR-132 mimics and occludin si-RNA under HG condition. Protein levels were normalized to GAPDH. D. Western blot analysis of p-JAK2 and p-STAT3 of ARPE-19 cells co-transfected with miR-132 mimics and/or occludin overexpression vectors under HG condition. Protein levels were normalized to GAPDH. Data are presented as the mean \pm standard error(SD) $(n=3) . * p<0.05, * * p<0.01$.

demonstrated that overexpression of occludin elevated the decreased level of occludin and lowered the increased cell permeability caused by miR-132 mimics (Fig. 5A-B). Additionally, we performed Western blot to evaluate the related molecules regulated by occludin, and found compared with $\mathrm{NC}$ mimics, both p-JAK2 and pSTAT3 were upregulated by miR-132 mimics under HG condition (Fig. 5C-D), and this upregulation was further enhanced by si-occludin and reversed by occludin overexpression. The results indicated that miR-132 activated the JAK2/STAT3 signaling pathway through occludin in ARPE-19 cells under HG condition.

\section{Discussion}

As a main cause of visual disability, DR mainly results from abnormal retinal blood vessels [3, 30]. Diabetic macular edema (DME) is the most common cause of central vision loss in diabetics [31]. It leads to vasogenic edema and pathological retinal thickening and is triggered secondarily to the deterioration of the BRB and the consequent increase in extravasation of fluids [32]. The $\mathrm{BRB}$, comprising the retinal vasculature and pigment epithelium, protects and allows the retina to regulate its extracellular chemical composition. Retinal capillaries form the inner BRB and it features endothelial cells harboring tight junctions that are almost impermeable to protein transport and help maintain the BRB, whose impairment could cause increased vascular permeability [33].

MiRNAs are a class of endogenous, small non-coding RNAs with 20-23 nucleotides long that are regulators of genes involving in the post-transcriptional regulation process [34]. They regulate gene expression by binding to the 3'UTR of the mRNAs, which in turn lead to translation inhibition. Aberrantly expressed miRNAs associated with cellular dysfunction in early DR as well as their targets were identified, indicating that miRNAs 
represent potential effective DR therapeutic targets [13]; among these, miR-132 was a potential DR-related miRNA, a result that needs further validation. The expression of miR-132 was increased by HG treatment in bone marrow stem cells [35]. On the contrary, the expression of miR-132 was significantly decreased in HG-treated vascular smooth muscle cells (VSMCs) [36]. In this study, we explored the function and downstream molecular mechanism of miR-132 in an in vitro cell model of DR and showed that it promotes cell mobility and permeability by regulating occludin and activating the JAK/STAT3 pathway.

Occludin is a protein encoded by the OCLN gene [37], the knockout of which conferred morphological stability of epithelial tissues, chronic inflammation, hyperplasia in the gastric epithelium and other effects [38, 39], suggesting that its function is quite complicated. The JAK/ STAT3 signaling pathway is involved in many processes including cell division, immunity, cell death and tumour formation [37]. Studies also showed that the JAK2/ STAT5 axis may partially mediate retinal vascular endothelial cell tolerance to hypoxia, indicating that it played a critical role during retinal angiogenesis. It is of much significance to endothelial cell survival to maintain the inappropriate retinal neovascularization common in DR during tissue hypoxia [40]. In our study, we for the first time revealed occludin is the target of miR-132. Additionally, to our knowledge there is at present no research on the JAK/STAT3 pathway affecting cell migration and leakage in DR.

Although this study reveals a new molecular mechanism of miR-132 regulation of DR based on a HG treated ARPE-19 cell model, much more needs to be done to deeply understand the pathological mechanism of DR. First, whether this molecular mechanism could be applied to an animal model needs further verification. Next, to better realize the potential of miRNA detection in DR, in future work, we will collect serum and vitreous tumour samples from DR patients and detect the expression of miR-132 in these samples to verify its potential as a serum marker. Besides, HG could induce the expression of miR-132 and reproduce DR-related phenotypes, meanwhile, miR-132 mimics alone without HG treatment cannot reproduce DR-related phenotypes, indicating that the miR-132 induction is not sufficient to reproduce DR-related phenotypes or even to suppress occludin and the co-existence of HG seems necessary for both and may have additional unknown effects, which needs further investigation. In conclusion, we for the first time revealed that the upregulation of miR-132 in ARPE-19 cells could enhance cell migration and permeability, and our findings suggested that the modulation of occludin targeting by miR-132 via the JAK/STAT3 pathway could represent novel effective DR-treatment strategies.

\section{Acknowledgements}

This work was supported by the National Natural Science Foundation of China (NO. 81660164), the Natural Science Foundation Key Program of Jiangxi Province (NO. 20171ACB20036), the Natural Science Foundation of Jiangxi Province (NO. 2015BAB205026 and NO. 2015BBG70171), the Scientific Research Program of the Second Affiliated Hospital of Nanchang University (NO. 2016YNZJ12001), the Scientific Research Program of Jiangxi Health and Family Planning Commission (NO. 2017A238) and Doctor Start-up Fund of the Second Affiliated Hospital of Nanchang University (NO. B1728\#).

\section{Ethics Approval and Consent to Participate}

Not Applicable. This article does not contain any studies with human participants or animals performed by any of the authors.

\section{Conflict of Interest Statement}

The authors declare that they have no conflict of interest.

\section{Authorship}

guarantor of integrity of the entire study: Shu-Hua $\mathrm{Fu}$ study concepts: Shu-Hua Fu study design: Shu-Hua Fu; Shan-Shan Wang definition of intellectual content: Xing Liao literature research: Fei Liu clinical studies: Shan-Shan Wang experimental studies: Shan-Shan Wang data acquisition: Qian Zhang data analysis: Shan-Shan Wang, Xing Liao statistical analysis: Jing-Jing Qiu, Qian Zhang manuscript preparation: Shan-Shan Wang, Fei Liu manuscript editing: Shu-Hua Fu manuscript review: Shu-Hua Fu 


\section{References}

1. Yau JW, Rogers SL, Kawasaki R, Lamoureux EL, Kowalski JW, et al. (2012) Global prevalence and major risk factors of diabetic retinopathy. Diabetes Care 35: 556-564.

2. Ting DS, Cheung GC, Wong TY (2016) Diabetic retinopathy: global prevalence, major risk factors, screening practices and public health challenges: a review. Clin Exp Ophthalmol 44: 260-277.

3. Antonetti DA, Klein R, Gardner TW (2012) Diabetic retinopathy. N Engl J Med 366: 1227-1239.

4. Cunha-Vaz JG, Travassos A (1984) Breakdown of the blood-retinal barriers and cystoid macular edema. Surv Ophthalmol 28 Suppl: 485-492.

5. Strauss $O$ (2005) The retinal pigment epithelium in visual function. Physiol Rev 85: 845-881.

6. Miyamoto N, de Kozak Y, Jeanny JC, Glotin A, Mascarelli F, et al. (2007) Placental growth factor-1 and epithelial haemato-retinal barrier breakdown: potential implication in the pathogenesis of diabetic retinopathy. Diabetologia 50: 461-470.

7. Silva PS, Cavallerano JD, Sun JK, Aiello LM, Aiello LP (2010) Effect of systemic medications on onset and progression of diabetic retinopathy. Nat Rev Endocrinol 6: 494-508.

8. Wang B, Atherton P, Patel R, Manning G,Donnelly R (2010) Antiangiogenic effects and transcriptional regulation of pigment epithelium-derived factor in diabetic retinopathy. Microvasc Res 80: 31-36.

9. Krick TW, Bressler NM (2018) Recent clinically relevant highlights from the Diabetic Retinopathy Clinical Research Network. Curr Opin Ophthalmol 29: 199-205.

10. Barot M, Gokulgandhi MR, Patel S, Mitra AK (2013) Microvascular complications and diabetic retinopathy: recent advances and future implications. Future Med Chem 5: 301-314.

11. Lynch SK, Abramoff MD (2017) Diabetic retinopathy is a neurodegenerative disorder. Vision Res 139: 101-107.

12. Wu JH, Gao Y, Ren AJ, Zhao SH, Zhong M, et al. (2012) Altered microRNA expression profiles in retinas with diabetic retinopathy. Ophthalmic Res 47: 195-201.

13. Kovacs B, Lumayag S, Cowan C, Xu S (2011) MicroRNAs in early diabetic retinopathy in streptozotocininduced diabetic rats. Invest Ophthalmol Vis Sci 52: 4402 4409.

14. McArthur K, Feng B, Wu Y, Chen S, Chakrabarti S (2011) MicroRNA-200b regulates vascular endothelial growth factor-mediated alterations in diabetic retinopathy. Diabetes 60: 1314-1323.

15. Kowluru RA, Koppolu P, Chakrabarti S,Chen S (2003) Diabetes-induced activation of nuclear transcriptional factor in the retina, and its inhibition by antioxidants. Free Radic Res 37: 1169-1180.

16. Beltrami C, Simpson K, Jesky M, Wonnacott A, Carrington C, et al. (2018) Association of elevated urinary
miR-126, miR-155, and miR-29b with diabetic kidney disease. Am J Pathol 188: 1982-1992.

17. $\mathrm{Hu} \mathrm{W}$, Jiang A, Liang J, Meng H, Chang B, et al. (2008) Expression of VLDLR in the retina and evolution of subretinal neovascularization in the knockout mouse model's retinal angiomatous proliferation. Invest Ophthalmol Vis Sci 49: 407-415.

18. Ting DS, Tan KA, Phua V, Tan GS, Wong CW, et al. (2016) Biomarkers of diabetic retinopathy. Curr Diab Rep 16: 125.

19. Murata M, Osanai M, Takasawa A, Takasawa K, Aoyama $\mathrm{T}$, et al. (2018) Occludin induces microvillus formation via phosphorylation of ezrin in a mouse hepatic cell line. Exp Cell Res 366: 172-180.

20. Zhu Y, Chen P, Gao Y, Ta N, Zhang Y, et al. (2018) MEG3 activated by vitamin D inhibits colorectal cancer cells proliferation and migration via regulating clusterin. EBioMedicine 30: 148-157.

21. Martin TA, Mason MD, Jiang WG (2011) Tight junctions in cancer metastasis. Front Biosci (Landmark Ed) 16: 898-936.

22. Peng S, Gan G, Rao VS, Adelman RA, Rizzolo LJ (2012) Effects of proinflammatory cytokines on the claudin-19 rich tight junctions of human retinal pigment epithelium. Invest Ophthalmol Vis Sci 53: 5016-5028.

23. Antonetti DA, Barber AJ, Khin S, Lieth E, Tarbell JM, et al. (1998) Vascular permeability in experimental diabetes is associated with reduced endothelial occludin content: vascular endothelial growth factor decreases occludin in retinal endothelial cells. Penn State Retina Research Group. Diabetes 47: 1953-1959.

24. Gardner TW (1995) Histamine, ZO-1 and increased blood-retinal barrier permeability in diabetic retinopathy. Trans Am Ophthalmol Soc 93: 583-621.

25. Kim JH, Jun HO, Yu YS, Kim KW (2010) Inhibition of protein kinase $\mathrm{C}$ delta attenuates blood-retinal barrier breakdown in diabetic retinopathy. Am J Pathol 176: $1517-1524$.

26. Lang GE (2012) Diabetic macular edema. Ophthalmologica 227 Suppl 1: 21-29.

27. Tien T, Barrette KF, Chronopoulos A, Roy S (2013) Effects of high glucose-induced Cx43 downregulation on occludin and ZO-1 expression and tight junction barrier function in retinal endothelial cells. Invest Ophthalmol Vis Sci 54: 6518-6525.

28. Xue J, Liao L, Yin F, Kuang H, Zhou X, et al. (2018) LncRNA AB073614 induces epithelial- mesenchymal transition of colorectal cancer cells via regulating the JAK/STAT3 pathway. Cancer Biomark 21: 849-858.

29. Wu H, Rahman HNA, Dong $\mathrm{Y}$, Liu X, Lee $\mathrm{Y}$, et al. (2018) Epsin deficiency promotes lymphangiogenesis through regulation of VEGFR3 degradation in diabetes. $J$ Clin Invest 128: 4025-4043.

30. Fu H, Tie Y, Xu C, Zhang Z, Zhu J, et al. (2005) 
Identification of human fetal liver miRNAs by a novel method. FEBS Lett 579: 3849-3854.

31. Mysona BA, Matragoon S, Stephens M, Mohamed IN, Farooq A, et al. (2015) Imbalance of the nerve growth factor and its precursor as a potential biomarker for diabetic retinopathy. Biomed Res Int 2015: 571456.

32. Moss SE, Klein R, Klein BE (1998) The 14-year incidence of visual loss in a diabetic population. Ophthalmology 105: 998-1003.

33. Scholl S, Augustin A, Loewenstein A, Rizzo S, Kupperman B (2011) General pathophysiology of macular edema. Eur J Ophthalmol 21 Suppl 6: S10-S19.

34. Ciulla TA, Amador AG, Zinman B (2003) Diabetic retinopathy and diabetic macular edema: pathophysiology, screening, and novel therapies. Diabetes Care 26: 2653 2664.

35. Kim YS, Kang HJ, Hong MH, Kang WS, Choe N, et al. (2014) Angiopoietin-like 4 is involved in the poor angiogenic potential of high glucose-insulted bone marrow stem cells. Korean Circ J 44: 177-183.
36. Xu Q, Liang Y, Liu XJ, Zhang CM, Liu XQ, et al. (2019) miR132 inhibits high glucoseinduced vascular smooth muscle cell proliferation and migration by targeting E2F5. Mol Med Rep 20: 2012-2020.

37. Yang Q, Li W, She H, Dou J, Duong DM, et al. (2015) Stress induces p38 MAPK-mediated phosphorylation and inhibition of Drosha-dependent cell survival. Mol Cell 57: 721-734.

38. Ando-Akatsuka Y, Saitou M, Hirase T, Kishi M, Sakakibara A, et al. (1996) Interspecies diversity of the occludin sequence: cDNA cloning of human, mouse, dog, and rat-kangaroo homologues. J Cell Biol 133: 43-47.

39. Saitou M, Furuse M, Sasaki H, Schulzke JD, Fromm M, et al. (2000) Complex phenotype of mice lacking occludin, a component of tight junction strands. Mol Biol Cell 11: 4131-4142.

40. Schulzke JD, Gitter AH, Mankertz J, Spiegel S, Seidler U, et al. (2005) Epithelial transport and barrier function in occludin-deficient mice. Biochim Biophys Acta 1669: 3442. 